\title{
A narrative review of targeted therapy in meningioma, pituitary adenoma, and craniopharyngioma of the skull base
}

\author{
Nina L. Martinez ${ }^{1 \wedge}$, Omaditya Khanna ${ }^{2}$, Christopher J. Farrell ${ }^{2}$ \\ ${ }^{1}$ Department of Neurology, Thomas Jefferson University, Philadelphia, PA, USA; ${ }^{2}$ Department of Neurological Surgery, Thomas Jefferson \\ University, Philadelphia, PA, USA \\ Contributions: (I) Conception and design: All authors; (II) Administrative support: All authors; (III) Provision of study materials or patients: All \\ authors; (IV) Collection and assembly of data: All authors; (V) Data analysis and interpretation: All authors; (VI) Manuscript writing: All authors; (VII) \\ Final approval of manuscript: All authors. \\ Correspondence to: Nina L. Martinez, MD. Thomas Jefferson University, 909 Walnut St, $2^{\text {nd }}$ Floor, Philadelphia, PA 19107, USA. \\ Email: nina.martinez@jefferson.edu.
}

\begin{abstract}
Management of solid tumors involving the skull base are primarily managed with surgery and radiation, though proximity to important vascular and neuroanatomic structures often limit the extent of resection and permissible radiation dose. Meningiomas are the most common primary brain tumor in adults, and although the majority of skull base meningiomas are low-grade, their location in proximity to critical anatomical structures precludes aggressive surgical resection, and larger tumors are often resistant to radiation treatment. In patients with clinically aggressive, unresectable meningiomas, several molecular biomarkers of angiogenesis, as well as genetic mutations (SMO, AKT1, PIK3CA, KLF4, POLR2, SMARCE1, and TRAF7), have been shown to play a crucial role in the pathophysiology of these tumors. Pituitary adenomas are commonly slow growing tumors that are amenable to surgical resection, but tumors with higher Ki67 proliferative indices are associated with an increased risk of relapse and resistance to standard therapies. Chemotherapeutic agents and checkpoint inhibitors have been trialed, albeit with limited success, to treat these aggressive pituitary adenomas. Craniopharyngiomas are categorized as adamantinomatous and papillary subtypes, each with unique molecular mechanisms that drive pathogenesis of these tumors, and have introduced the possibility that targeted therapies may be developed for improved neurologic and endocrinological outcomes. Skull base tumors that exhibit recurrence despite surgical resection and radiation treatment pose a unique challenge, and systemic agents offer a non-invasive option of treating tumors that are refractory to conventional approaches. Recent insights into the molecular aberrations that elucidate the pathophysiology of these difficult-to-treat tumors have provided potential therapeutic targets for drug delivery. In this review, the authors discuss promising therapies and current knowledge gaps needed for the development of effective targeted agents for meningioma, pituitary adenoma, and craniopharyngioma.
\end{abstract}

Keywords: Chemotherapy; immunotherapy; cranial base tumor; vascular endothelial growth factor (VEGF); temozolomide; $B R A F$

Submitted Jun 10, 2020. Accepted for publication Jul 17, 2020.

doi: $10.21037 /$ cco-20-168

View this article at: http://dx.doi.org/10.21037/cco-20-168

$\wedge$ ORCID: 0000-0002-9031-4518. 


\section{Introduction}

Tumors of the skull base region are frequently slowgrowing and benign, though intervention is often pursued when symptoms occur. These can be challenging to manage and often require a multidisciplinary approach to optimize outcome and quality of care. Surgery is central to treatment; however, complete resection may be precluded by nearby critical vasculature and neuroanatomic structures such as cranial nerves and, in time, the residual tumor may grow. Radiation is beneficial and frequently used in unresectable or residual tumors, but its use is also limited in multiply recurrent tumors.

Systemic agents such as chemotherapy have been explored in the past and generally have been ineffective, though improved knowledge of tumor biology has led to promising developments in systemic options. In this review, we discuss the current molecular and genetic understanding of meningiomas, pituitary adenomas, and craniopharyngiomas as it relates to the development of targeted therapy. We present the following article in accordance with the NARRATIVE REVIEW reporting checklist (available at http://dx.doi.org/10.21037/cco-20-168).

\section{Methods}

A literature search of PubMed was performed to identify publications-including basic science research, clinical studies, and case reports-pertaining to skull base meningiomas, pituitary adenomas, and craniopharyngiomas that are resistant to standard surgical and radiation therapy. No publication limits were applied, and no inclusion or exclusion criteria were implemented for the literature search. The authors provide a narrative summary of the findings and implications of these studies, and how they pertain to improving the clinical care of our own patients with these pathologies.

\section{Meningiomas}

Meningiomas are the most common type of primary brain tumors in adults (1). The tumors originate from progenitor cells in the arachnoid layer of the meninges that envelop the brain and spinal cord and their clinical presentation is diverse: $30 \%$ present with seizures due to mass effect along the cerebral convexity or parasagittal region, and an equal percentage may present with symptoms such as impaired hearing, loss of smell, or visual changes from encroachment of the skull base (2). Meningioma behavior can be predicted partly by histological grade (3-5). The current World Health Organization (WHO) defines three grades which correspond to recurrence risk based on features including nuclear atypia, mitotic activity, invasion of adjacent structures, and presence of necrosis (6). Furthermore, each grade is subdivided into a number of subtypes, some of which have a predilection for the skull base. Approximately $80 \%$ of meningiomas are classified as benign (grade I) with a low rate of recurrence (7-20\%) (6-8). The remainder are classified as either atypical (grade II) or anaplastic (grade III) and are associated with significantly shorter recurrencefree intervals and higher mortality rates (40-70\%) (9-12).

Surgical intervention is often the first step in the management of large or symptomatic tumors. Gross total resection (GTR) is associated with better local control and progression-free survival compared to subtotal resection (STR), independent of grade $(13,14)$. However, complete removal is not always feasible due to a tumor's proximity to critical vessels or eloquent brain structures, which is commonly the case in the skull base region. In selected patients, particularly those with clinically aggressive, unresectable meningiomas, radiation can be employed in either adjuvant or primary settings in hopes of improving local control over observation alone. Both fractionated external beam radiation (EBRT) and stereotactic radiation (SRS) have been used, and the choice of delivery depends on the size of the target and tumor grade. SRS is a widely accepted technique for small grade I or II lesions, while EBRT is recommended for grade III meningiomas which require larger doses (50-60 Gy) to achieve local control $(15-17)$. Yet a small subset of these patients remains refractory to standard intervention; thus, numerous systemic agents have been explored, including hormone receptor inhibitors, somatostatin analogs, and conventional chemotherapy, with none demonstrating reproducible benefit (18-24). As molecular and genomic techniques advance, so does our understanding of tumor biology and potential targets for therapeutic intervention $(25,26)$.

Aberrations in cell signaling pathways can initiate downstream effects that contribute to the development of meningiomas. Clarifying which growth factors and downstream signaling pathways are critical to the pathogenesis can be challenging and continues to evolve. Vascular endothelial growth factor (VEGF) and plateletderived growth factor (PDGF) have been implicated in meningioma growth and are potential therapeutic targets.

Meningiomas are highly vascularized and express 

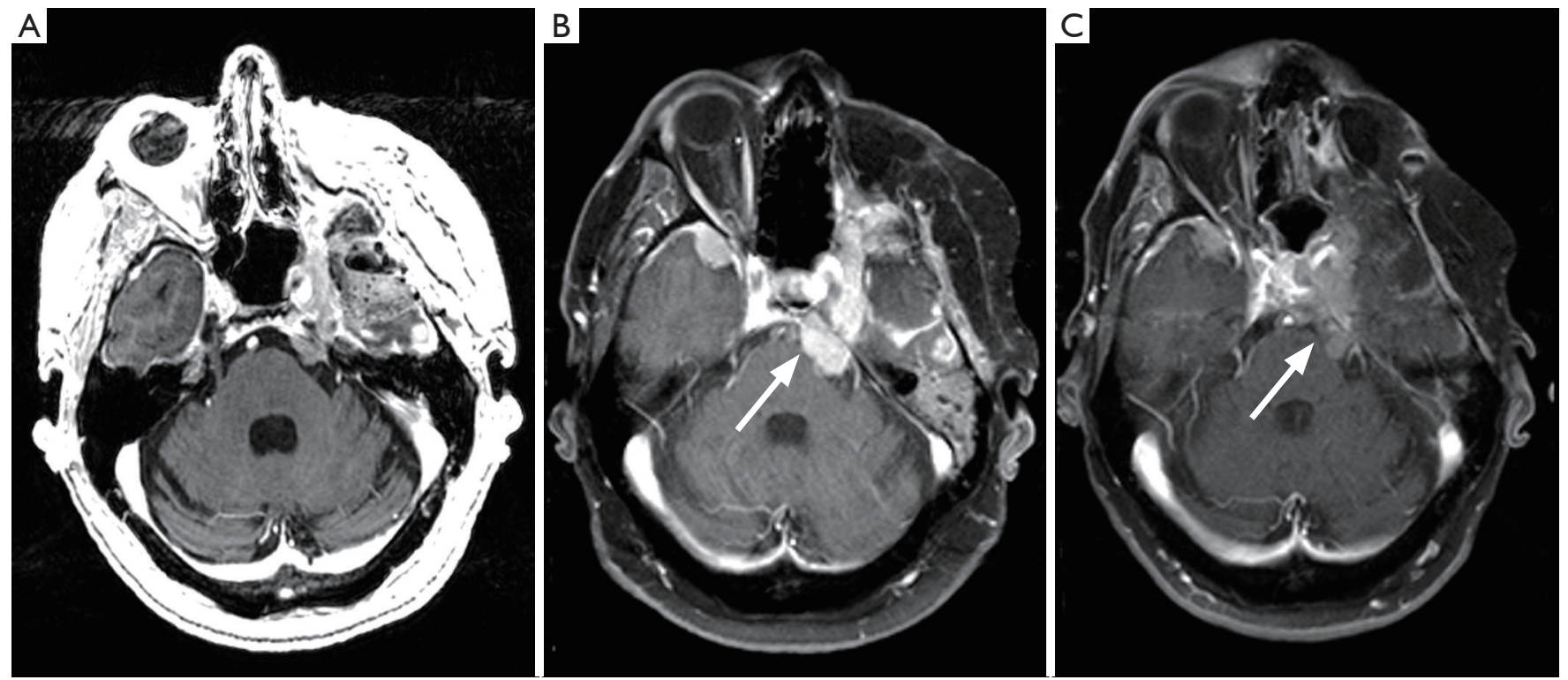

Figure 1 A 57-year-old man with multiply recurrent WHO grade II meningioma who had undergone three resections and three courses of radiation to multiple tumor sites since initial diagnosis in 2013. (A) Surveillance MRI one day after resection of a left temporal recurrence. (B) Baseline brain MRI prior to initiation of bevacizumab (10 mg/kg intravenously every 14 days) and everolimus (10 mg orally daily). Note interval growth of left cerebellopontine meningioma (arrow). (C) Surveillance scan after one year on combination therapy. The cerebellopontine meningioma has decreased somewhat in size, and no new tumors had developed in the interim.

angiogenic factors including VEGF. Meningioma extracts have been shown to induce endothelial chemotaxis and capillary-like tube formation in vitro, and positive correlation between VEGF content and meningioma grade has been demonstrated though not with vascularity or invasiveness (27-29). VEGF is also involved in the development of peritumoral edema, which is one cause of morbidity in high grade meningiomas (30). Antibodies against VEGF may be effective in controlling symptomatic edema and tumor growth. In one retrospective study, bevacizumab (a humanized monoclonal antibody against VEGF-A) was most effective in slowing tumor growth and reducing peritumoral edema when compared to other systemic therapies such as cytotoxic chemotherapy, somatostatin analogues, and tyrosine kinase inhibitors (31). A phase 2 single-arm study evaluating the effect of bevacizumab in combination with everolimus, an inhibitor of mammalian target of rapamycin (mTOR), in refractory, progressive intracranial meningioma demonstrated favorable results (Figure 1) (32). Though the study was terminated early due to slow accrual, $88 \%$ [15/17] of the patients who received study treatment had stable disease; six of these 15 patients had stable disease beyond a year. Median progression-free survival (mPFS) was higher in patients with grade II and III meningiomas (22 months) compared to grade I (17.5 months). The treatment was fairly well tolerated, as grade 3 and higher toxicities were uncommon. However, the small sample size was small and the results require confirmation with larger trials. Results of a prospective phase 2 trial of single-agent bevacizumab in patients with recurrent or progressive meningiomas closed to accrual are forthcoming (NCT01125046).

There is also evidence suggesting the importance of PDGF in meningioma cell growth and provide rationale for testing PDGF inhibitors in patients with progressive or recurrent meningiomas (33-36). The therapeutic potential of imatinib mesylate, a PDGF receptor (PDGFR) inhibitor, was evaluated in a phase 2 study conducted by the North American Brain Tumor Consortium (NABTC 01-08). The oral agent was generally well tolerated, though the efficacy data were disappointing. Of the 19 patients evaluable for response, 10 patients progressed at the first scan and 9 had stable disease (37). In another phase 2 study, the combination of imatinib and hydroxyurea was modest at best in that only $30 \%$ [4/13] of patients with grade II or III meningiomas achieved stable disease longer than 6 months (38). Thus, there is increasing attention to the development of novel and multi-targeted agents. Sunitinib 
is a small molecule multi-targeted receptor tyrosine kinase (RTK) inhibitor that targets VEGF receptor (VEGFR) and platelet-derived growth factor receptor (PDGFR), and appears to be active even in heavily pre-treated patients with high-grade meningioma; however, toxicity is a concern. In a phase 2 trial, $42 \%$ were alive and without disease progression at 6 months (PFS-6), though 60\% experienced a severe adverse event (39). Vatalanib, another multitargeted RTK inhibitor, demonstrated a PFS-6 of 54.4\% for grade II and III meningiomas combined, though since the study was powered for grade I meningiomas it is unclear whether the phase 2 trial was positive and remains a major study limitation (40).

In contrast to the amount of work focused on molecular targets, efforts to understand genomic drivers of meningioma growth have increased. The most frequent genetic alteration in meningioma tumorigenesis involve mutations or loss of heterozygosity of the tumor suppressor gene NF2, which encodes the protein merlin and is located on chromosome 22. Individuals affected by germline mutations of NF2 develop neurofibromatosis type 2 (NF2) and are predisposed to developing meningiomas among other benign tumors such as schwannomas and ependymomas. Although sporadic meningiomas are by far more common, they too are often driven by this mutation, which is associated with fibrous or transitional (WHO grade I) and are more frequently found along the cerebral convexities than skull base (41). Merlin is involved in key signaling pathways such as mTOR that regulate cell shape, growth, and adhesion. In merlin-deficient meningioma cells, cell proliferation and survival are increased as a consequence of deregulated downstream effects of mammalian target of rapamycin complex 2 (mTORC1) and mTORC2 $(42,43)$. In pre-clinical studies, mTORC1 inhibitors such as everolimus and temsirolimus, and the dual mTORC1 and mTORC2 inhibitor vistusertib (AZD2014) demonstrate promising activity against the growth of meningioma cells and have warranted further study in humans. In a phase II trial, the combination of bevacizumab $(10 \mathrm{mg} / \mathrm{kg}$ IV every 14 days) and everolimus (10 mg PO daily) produced stable disease (SD) in 15/17 (88\%) of patients; 6 patients had SD for over 12 months (32). The efficacy of vistusertib in patients with recurrent, progressive, or symptomatic meningiomas is currently under investigation in two phase II trials (NCT03071874, NCT02831257). In the single-center, open-label study of patients with NF2 and progressive or symptomatic meningiomas, 18 patients received vistusertib $125 \mathrm{mg}$ BID for two consecutive days each week. Radiographic response, defined as a $20 \%$ decrease from baseline, was seen in $5-10 \%$ of schwannomas and meningiomas, though $17 \%$ of target meningiomas progressed. Furthermore, seven patients (39\%) elected to discontinue treatment due to intolerable side effects including fatigue, anorexia, nausea, vomiting, rash, and mucositis (Plotkin S, 2018, unpublished data). The relationship between tumor regression and activation of TORC1/2 pathways has yet to be clarified.

Next-generation sequencing has made it possible to identify several recurrent mutations in genes other than NF2, including SMO, AKT1, PIK3CA, KLF4, POLR2, SMARCE1, and TRAF7, which are mutually exclusive with NF2. Meningiomas harboring these mutations also demonstrate mutant-specific locations in the skull base and histological subtypes (44-49). The majority of $S M O, A K T 1$, and PIK3CA-mutant meningiomas occur in the anterior skull base and are strongly associated with the grade I meningothelial subtype (50). Smoothened (Smo), which is encoded by $S M O$, is a transmembrane protein integral to the Hedgehog $(\mathrm{Hh})$ signaling pathway and plays a key role in embryogenesis. Inhibition of Smo by the smallmolecule drug vismodegib is currently under investigation in combination with a novel FAK inhibitor GSK2256098 (NCT02523014).

$A K T 1$ encodes the enzyme RAS-alpha serine/ threonine-protein kinase and is activated through the phosphatidylinositol-3 kinase (PI3K)/Akt/mTOR pathway to suppress apoptosis. Grade I meningiomas which harbor activating mutations of $A K T^{E 17 K}$ are characterized by shorter time to recurrence compared to $A K T 1$ wildtype meningiomas (51). A multicohort basket study of patients with $A K T 1$-mutant solid tumors demonstrated that treatment with the pan-Akt inhibitor AZD5363 is safe and effective (52). Although patients with meningiomas were not included in this study, Weller et al. reported an encouraging case in which a patient with multiply recurrent, meningothelial meningioma metastatic to the lung achieved stable disease of multiple tumor sites after 17 months of salvage AZD5363 (given $480 \mathrm{mg}$ twice daily in a four days on/three days off schedule). Further exploration of targeted therapy in this subgroup of meningiomas is warranted (53).

Approximately $7 \%$ of $N F 2$ wild-type meningiomas involve PIK3CA, which is mutually exclusive with $S M O$ and $A K T 1$, and approximately $25 \%$ harbor mutations in tumor necrosis factor receptor-associated factor 7 (TRAF7), which is involved in ubiquitination and can co-occur with $A K T 1$ or Krüppel-like factor 4 (KLF4) gene $(48,54)$. Both TRAF7 
and KLF4 occur in secretory meningioma (WHO grade I) and tend to be located in the petroclival or midline skull base. Currently the tumorigenic roles of TRAF7 or KLF4 are unclear, and warrant further study before effective targeted agents can be developed.

In contrast to grade I meningiomas, grade II and III meningiomas exhibit fewer somatic targetable mutations such as $S M O, A K T 1$, or PIK3CA. Instead, higher grade meningiomas are more likely to demonstrate high mutational burden, high-frequency microsatellite instability (MSI-H), and mismatch repair (MMR) deficiency from copy number alterations. Compared to specific driver alterations, these are more challenging to target selectively and may respond more favorably to a broader approach. The investigation of immunotherapy such as programmed death-1 receptor (PD-1) blockade is supported by a number of observations of high-grade meningiomas: a number of mutations are predicted to be immunogenic, expression of PD-1 and its ligand (PD-L1) is increased, and the microenvironment of meningiomas contain a selected, antigen-experienced effector T-cell population enriched by those expressing markers of an exhausted phenotype (44,55-57). Several phase II clinical trials are actively recruiting to assess the efficacy of checkpoint inhibitors such as pembrolizumab, nivolumab, and ipilimumab in recurrent or residual highgrade meningiomas (NCT02648997, NCT03279692, NCT03604978, NCT03173950).

While surgery and radiation remain central to the initial management of enlarging, symptomatic meningiomas, improved understanding of the underlying tumor biology has refined the options for systemic therapy. The agent of choice will likely depend on molecular and genetic profiling, as the response of high-grade meningiomas to highly selective agents is not expected to be as favorable as a subset of non-NF2-mutant WHO grade I meningiomas might. Regardless, early data are promising and give hope to controlling the disease in patients who have exhausted all other options.

\section{Pituitary adenomas (PA)}

PA account for approximately $10-20 \%$ of primary intracranial tumors and may cause clinical symptoms either due to hormonal hypersecretion or compression of surrounding structures including the optic nerves, chiasm, and cavernous sinuses. The majority of these tumors are benign and slow growing and are highly treatable with standard therapies including surgical resection and adjuvant radiation therapy. While surgical resection via either a transsphenoidal or transcranial route continues to be the mainstay of treatment for most symptomatic pituitary adenomas, the extent of resection is highly dependent on a number of factors including tumor size, consistency, and invasion into the parasellar and suprasellar areas. Following subtotal resection, $50-60 \%$ of adenomas will continue to progress and even after apparent gross total resection, tumor recurrence may occur in up to $30 \%$ of cases with longterm follow-up (58-62). Adjuvant radiation therapy is often used for the treatment of residual and recurrent pituitary adenomas and is typically delivered using stereotactic radiosurgery (SRS), conventional conformal radiotherapy, or fractionated stereotactic radiotherapy (FSRT). The goals of radiation therapy are typically to achieve tumor control for non-functioning adenomas (NFPA) and tumor control with endocrine remission for hormone-secreting functional adenomas. SRS is the most common modality used to treat recurrent pituitary adenomas with tumor control rates of $94-100 \%$ at 5 years and $76-87 \%$ at 10 years of follow-up for NFPA. SRS is typically delivered in a single session with a marginal dose of 12-18 Gy. Post-radiation neurological deficits and hypopituitarism following SRS may result in up to $7 \%$ and $39 \%$ of patients, respectively (63). Additional potential complications of radiation therapy include symptomatic radiation necrosis, secondary neoplasms, and radiation-induced vasculopathy. Radiation therapy also plays an important role in inducing hormonal remission for recurrent functional adenomas resistant to medical therapies and not amenable to further surgery. Endocrine remission rates for ACTH-secreting adenomas with SRS range from $28-70 \%$ with higher radiation doses necessary to induce remission compared to those necessary for tumor control with NFPA.

A small subset of pituitary tumors is classified as aggressive pituitary adenomas (APA) based on their clinical presentation and resistance to standard surgical, radiation, and medical therapies (64). A clear definition for APA remains controversial, however, the most recent $4^{\text {th }}$ edition of the WHO Classification of Tumors of the Pituitary Gland (2017) recognizes the importance of increased mitotic count, high Ki67 proliferative index ( $>3 \%)$, and tumor invasion as pathologic markers of clinically aggressive adenomas with an increased risk of relapse and resistance to standard therapies (Figure 2) $(65,66)$. Additionally, several subtypes of pituitary neuroendocrine tumors with a high probability of recurrence have been designated including silent corticotroph adenomas, sparsely granulated somatotroph 

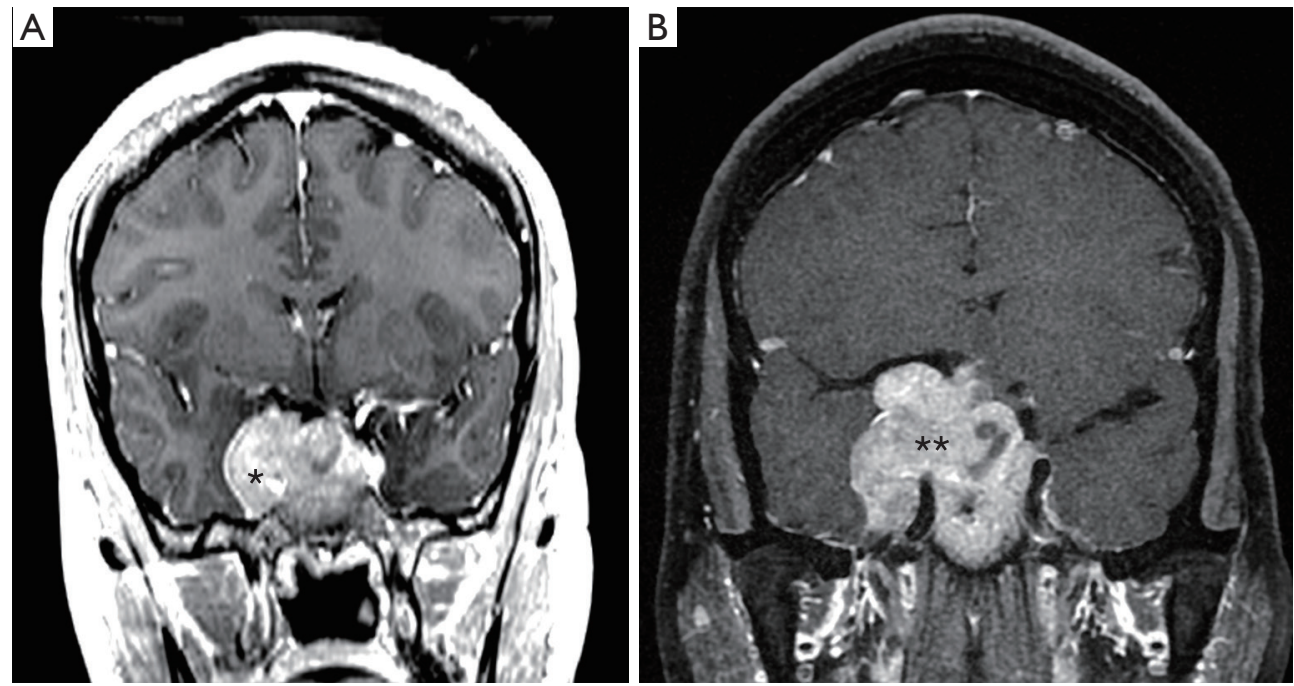

Figure 2 Hormone-secreting pituitary macroadenoma, refractory to multi-modality therapy. (A) Coronal MRI with contrast demonstrating a prolactin-secreting pituitary macroadenoma with right cavernous sinus invasion $\left(^{*}\right)$. (B) Despite dopamine agonist therapy, fractionated stereotactic radiotherapy and transsphenoidal surgical resection, the macroadenoma continued to progress encasing the right carotid artery ${ }^{* *}$ and compressing the optic chiasm (arrow) resulting in visual loss.

adenomas, lactotroph adenomas in med, Crooke's cell adenoma, and plurihormonal Pit-1-positive adenomas. This new pathologic classification should enable more accurate tumor subtyping and improved prediction of aggressive clinical behavior to better assess and guide our therapies. The 2017 WHO guidelines continue to define pituitary carcinomas by the presence of non-contiguous craniospinal or systemic metastases as pituitary carcinomas and adenomas are histologically indistinguishable (Figure 3). Fortunately, pituitary carcinomas remain rare, accounting for only $0.2 \%$ of pituitary tumors, but are associated with 1-year mortality rates of up to $66 \%$ (67). In a 2016 European Society of Endocrinology (ESE) survey of treatment of aggressive pituitary tumors, the mortality rate for APA nearly approaches that of pituitary carcinomas, demonstrating the need for improved chemotherapy and targeted molecular therapies for these challenging tumors $(68,69)$.

Recently, significant advances have been made in understanding the genetic landscape of pituitary adenoma tumorigenesis. The primary pathomechanism of pituitary adenomas appears to involve alterations in cell-cycle regulation and growth factor signaling due to epigenetic changes. Somatic and germline mutations occur more rarely with growth-hormone and ACTH-secreting adenomas carrying somatic mutations in the GNAS and USP8 genes, respectively (70). Mutations in USP48 and $B R A F$ have also been identified in USP8-negative Cushing's disease patients (71). More rarely, germline mutations occur in association with familial tumor syndromes including multiple endocrine neoplasia type 1 (MEN1) and type 4 (MEN4) syndromes, Carney complex, and McCune-Albright syndrome (70). While the identification of germline and somatic genetic defects has contributed to our understanding of PA pathogenesis, the exact molecular mechanisms leading to the majority of PA remains to be fully elucidated and the distinction of these molecular subgroups has remained of limited clinical relevance in terms of treatment.

Given the complex genomic landscape of PA, a wide variety of therapies have been tried for aggressive pituitary adenomas and carcinomas, mostly with poor results. To date, the most commonly utilized therapies have been alkylating chemotherapeutics previously approved for other central nervous system malignancies. In 2006, a series of case reports described regression of aggressive pituitary adenomas following administration of temozolomide (TMZ) monotherapy $(72,73)$. More recently, McCormack and colleagues reported the results of a European Society of Endocrinology (ESE) survey on the treatment of APA and PC (69). The survey included 157 patients treated with TMZ as first-line chemotherapy reported that overall 37\% of patients demonstrated a radiographic response including $6 \%$ with complete regression. The majority of these patients were administered TMZ according to the standard 

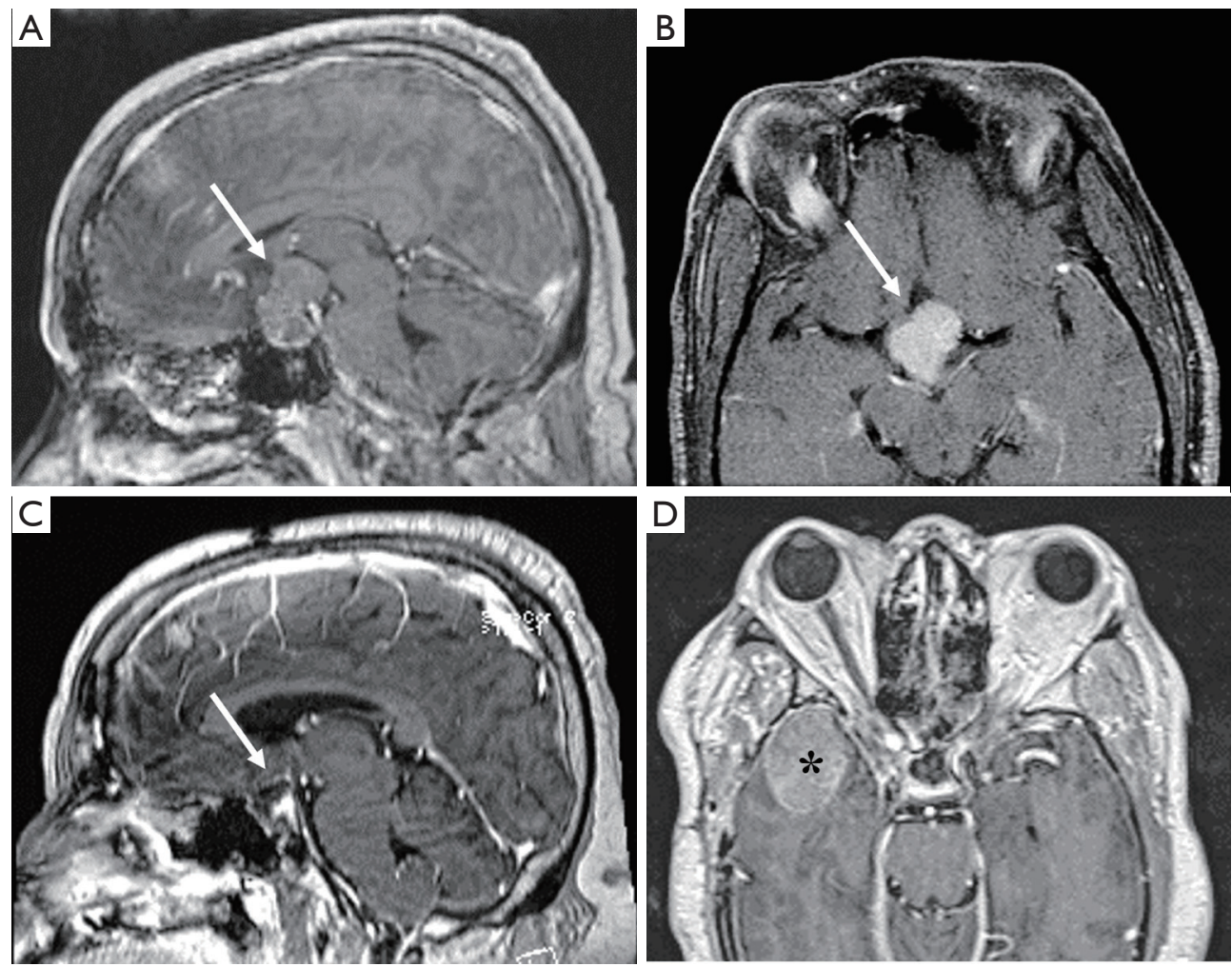

Figure 3 A 63-year-old man presented with worsening diabetes mellitus and visual loss. Laboratory evaluation revealed an elevated growth hormone $(\mathrm{GH})$ of $53 \mathrm{ng} / \mathrm{mL}(<10.0)$ and a pituitary macroadenoma (arrow) with suprasellar extension on sagittal (A) and axial (B) MRI. He underwent a right craniotomy for near-gross total resection of the mass followed by stereotactic radiosurgery with his postoperative GH decreased to $3.7 \mathrm{ng} / \mathrm{mL}$. Three years after treatment, laboratory and imaging surveillance revealed his GH increased to 134 and MRI showed no sellar recurrence (C) but non-contiguous growth of his pituitary tumor (D) along the right sphenoid wing $\left(^{*}\right)$ consistent with pituitary carcinoma.

"Stupp" protocol used for glioblastoma (TMZ $75 \mathrm{mg} / \mathrm{m}^{2}$ daily, followed by TMZ $150-200 \mathrm{mg} / \mathrm{m}^{2}$ daily for five days every 28 days). The median duration of treatment was 9 months and the maximal radiographic response occurred within 3 months in $23 \%$ of patients and within 6 months in $59 \%$ of patients (74). Hormonally functioning tumors responded better to TMZ than NFPA, and similar to the responses seen with glioblastoma, lower activity levels of the DNA repair enzyme 0(6)-methylguanine methyltransferase (MGMT) were associated with improved clinical response (69). On the basis of these results, the ESE guidelines for the management of APA and PC currently recommend use of TMZ monotherapy as first-line chemotherapy for these tumors following documented tumor growth (64). The ESE recommends that radiographic response be evaluated after three cycles of TMZ and therapy continued for at least six months for tumors demonstrating clinical response or stability. Little data is available on the long-term treatment of APA and PC, but TMZ monotherapy is often continued until progression is observed.

There is limited data to guide second-line therapies for APA and PC that continue to progress following TMZ administration. Preclinical and clinical studies have identified several pituitary adenoma-intrinsic targets for therapy including growth factors and their receptors and intracellular signaling pathways. Inhibition of VEGF has been reported in 13 cases, with 9 cases showing apparent clinical response to either anti-VEGF monotherapy or combination therapy (TMZ, pasireotide, radiotherapy) (68). Markers of tumor angiogenesis, including VEGF expression, have been observed in APA compared to standard pituitary adenomas. Ortiz et al. described a patient with a silent corticotroph pituitary carcinoma who 
had progressed despite $T M Z$ administration treated with bevacizumab. After 26 months of bevacizumab treatment, stability of disease was observed on serial MRI and PET scan imaging (75). Wang et al. reported a $>90 \%$ tumor size reduction of a multiply recurrent aggressive growth hormone-producing adenoma following treatment with TMZ and the VEGFR-2 inhibitor apatinib (76). After two years of follow-up, they reported sustained biochemical growth hormone remission and no radiographic tumor recurrence. The relative contribution of apatinib to the clinical response remains uncertain, however. Based on these limited data, VEGF-targeted therapies should be considered in patients with progressive disease. The clinical relevance of increased VEGF-expression as a biomarker to predict response deserves further evaluation. The Raf/ MEK/ERK and PI3K/Akt/mTOR pathways have also been shown to be upregulated in pituitary tumors $(64,77)$. Inhibitors of these cascades demonstrate anti-tumor effects in vitro and in murine models of APA, however, limited success has been observed clinically with these agents. Everolimus, an oral mTOR inhibitor, has been employed as second- or third-line monotherapy in five patients without success $(64,78)$. The oncogenic V600E BRAF mutation has been detected in $16.5 \%$ of corticotroph adenomas but not in other types of pituitary adenomas. Treatment of murine corticotroph pituitary adenomas with the BRAF inhibitor vemurafenib reduced ACTH expression, suggesting a role for this inhibitor in Cushing's disease patients with BRAFmutated tumors $(68,71)$.

An additional emerging therapy for treatment refractory pituitary adenomas is the use of immunotherapy. A recent study by Wang et al. reported that PD-LI, a key predictive marker of immunotherapy response, is frequently expressed in functional pituitary adenomas with higher Ki-67 index (79). PD-L1 expression was detected in $59 \%$ of functional tumors compared to $34 \%$ of NFPA. Similarly, Mei et al. showed significantly higher levels of PDL1 expression and tumor infiltrating lymphocytes in functional tumors, further raising the possibility that checkpoint blockade immunotherapy may be effective in cases of functional adenomas refractory to conventional management (80). Currently, only a single case has been reported of a patient with a pituitary carcinoma, previously treated with TMZ and capecitabine, responding to treatment with a combination of the checkpoint inhibitors monoclonal antibodies nivolumab (anti-PD-1) and ipilimumab (anti-CTLA4). Following five cycles of therapy, the intracranial tumor volume decreased by $59 \%$ and the primary liver metastasis decreased by $92 \%$ (81). Plasma ACTH levels decreased from 45,550 to $66 \mathrm{pg} / \mathrm{mL}$. At six months of surveillance, no progression was observed. Importantly, clinical experience with immunotherapy suggests that normal pituitary cells are susceptible to checkpoint inhibitors with hypophysitis reported as a common side effect of therapy in $4-15 \%$ of patients, potentially related to ectopic expression of CTLA-4 on pituitary endocrine (82-84). While this expression may potentiate the anti-tumor immune response of checkpoint inhibitors, patients will need to be closely monitored for development of hypopituitarism.

In conclusion, following failure of conventional treatments including surgical resection by an expert neurosurgeon and maximal radiation therapy for aggressive pituitary adenomas and pituitary carcinomas, TMZ is currently recommended as first-line therapy. Secondand third-line options remain uncertain with limited data available to guide therapy, however, treatment with VEGF inhibitors and immune checkpoint inhibitors appear to hold promise and are currently being investigated in several clinical trials.

\section{Craniopharyngiomas}

Craniopharyngiomas are benign (WHO grade I) tumors that occur in the sellar and suprasellar region, arising from embryonic epithelium derived from Rathke's pouch (85). Craniopharyngiomas are relatively rare tumors with an incidence of 0.13 per 100,000-person years and account for $3 \%$ of all intracranial neoplasms (1). Craniopharyngiomas reliably occur in a bi-modal age distribution, most often presenting in pediatric patients between the age of $5-14$, with a second peak occurring in adults in their sixth to seventh decade of life (86). There are two subtypes of craniopharyngiomas, each with a predilection to occur in different patient populations: the adamantinomatous subtype most frequently affects pediatric patients, whereas papillary subtype predominantly occurs in adults (87). Radiographically, adamantinomatous craniopharyngiomas contain prominent cystic components and frequently exhibit calcifications, whereas the papillary subtype is more solid and rarely contains calcifications (88). Given their location in the suprasellar space, craniopharyngiomas may exert mass effect on nearby structures, and consequently can present with vision changes from compression of the 
optic apparatus, hormonal abnormalities secondary to hypothalamic-pituitary dysfunction, or headaches due to obstructive hydrocephalus (89).

Craniopharyngiomas are slow-growing tumors and diagnosis is often delayed until patients begin exhibiting symptoms due to compression of adjacent structures. The most common clinical symptoms at the time of diagnosis include headaches, visual impairment, and endocrine dysfunction (90). Pediatric patients are frequently found to have endocrinopathies at the time of diagnosis, most commonly growth hormone and gonadotrophin deficiency (90). Adults, on the other hand, more often present with headaches and visual field defects (91).

The treatment of craniopharyngiomas in both pediatric and adult patients typically involves surgical resection followed by adjuvant radiation therapy. Surgical resection serves to reduce tumor mass effect on the optic apparatus and brainstem as well as restore cerebrospinal flow to relieve hydrocephalus. Resection also provides tissue diagnosis for histopathology and molecular genotyping and decreases the overall tumor volume for subsequent radiation treatment if necessary (92). Surgical resection can be performed via a transcranial or endoscopic endonasal approach (93), both of which have shown to confer similar overall survival when combined with adjuvant radiation therapy (94-96). Radiation treatment has been shown to be an effective adjuvant treatment following subtotal tumor resection or at the time of tumor recurrence (97-99).

Surgical resection and radiation therapy provide excellent tumor control, with overall 10-year survival rates reported between $80-90 \%$, albeit local recurrence is common $(100,101)$. Even amongst patients with longterm progression free survival, there is a high prevalence of neurological and psychological morbidity. Patients with craniopharyngioma diagnosed in childhood, and their caregivers, report inferior quality of life metrics, and harbor long-term psychosocial impairment (102). Furthermore, pituitary insufficiency is present in a large portion of patients, and up to $75 \%$ of patients are rendered with panhypopituitarism, predisposing them to chronic morbidity from obesity, hyperlipidemia, diabetes, and cardiovascular disease (103). The neurocognitive and metabolic morbidity associated with treatment of craniopharyngiomas via aggressive surgical resection and subsequent radiation therapy underscores the need for research and development of novel drug therapies. In recent years, insights gained from the molecular mechanisms that drive pathogenesis of craniopharyngiomas have introduced the possibility that targeted therapies may developed for the treatment of these tumors.

\section{CTNNB1/B-catenin gene mutation in adamantinomatous craniopharyngioma}

A mutation of CTNNB1 resulting in the over-expression $\beta$-catenin, mediated via activation of the $W n t$ signaling pathway, has been implicated in the tumorigenesis of adamantinomatous craniopharyngiomas (104). $\beta$-catenin is a protein encoded by the CTNNB1, and is part of the cadherin protein complex that mediates cellular adhesion (105). $\beta$-catenin also comprises an important part of the $W n t$ signaling pathway, which mediates cellular proliferation. Aberrations in the $\beta$-catenin/Wnt signaling pathway have been implicated in the pathogenesis of breast, colorectal, and endometrial cancer (106). In its inactivated form, $\beta$-catenin is found in the cellular membrane, where it can be readily degraded by proteasomes. Mutations in the CTNNB1 has been shown to activate the $W n t$ pathway, which renders $\beta$-catenin resistant to degradation and instead mobilizes $\beta$-catenin to the nucleus of cells to promote tumor cell migration (107).

Sekine et al. performed DNA sequencing analysis on 16 patients who underwent resection of craniopharyngiomas and found that all ten adamantinomatous specimens harbored $\beta$-catenin gene mutations, which was not found in any of the papillary subtype samples (108). Immunohistological analysis revealed the cellular location of $\beta$-catenin, too, was significantly different between the two subtypes. The adamantinomatous subtype showed predominantly cytoplastic and nuclear expression of $\beta$-catenin, with lack of membranous staining, in contrast to papillary subtype specimens that exclusively harbored $\beta$-catenin in cellular membranes. Taken together, this study was the first to show that in addition to separate histologic findings between the two tumor subtypes, adamantinomatous craniopharyngiomas harbor a distinct genetic profile compared to papillary craniopharyngiomas, with different underlying mechanisms of tumorigenesis (Table 1).

The exon 3 location of the CTTNB1 encodes for the glycogen synthase kinase-3 (GSK-3) domain responsible for the phosphorylation of $\beta$-catenin, and mutations in this gene prevent the protein from effectively being marked for proteolysis. Goschzik et al. examined whether the CTTNB1/ $\beta$-catenin mutation at different phosphorylation sites 
Table 1 Clinical and genetic characteristics of adamantinomatous and papillary subtypes of craniopharyngioma

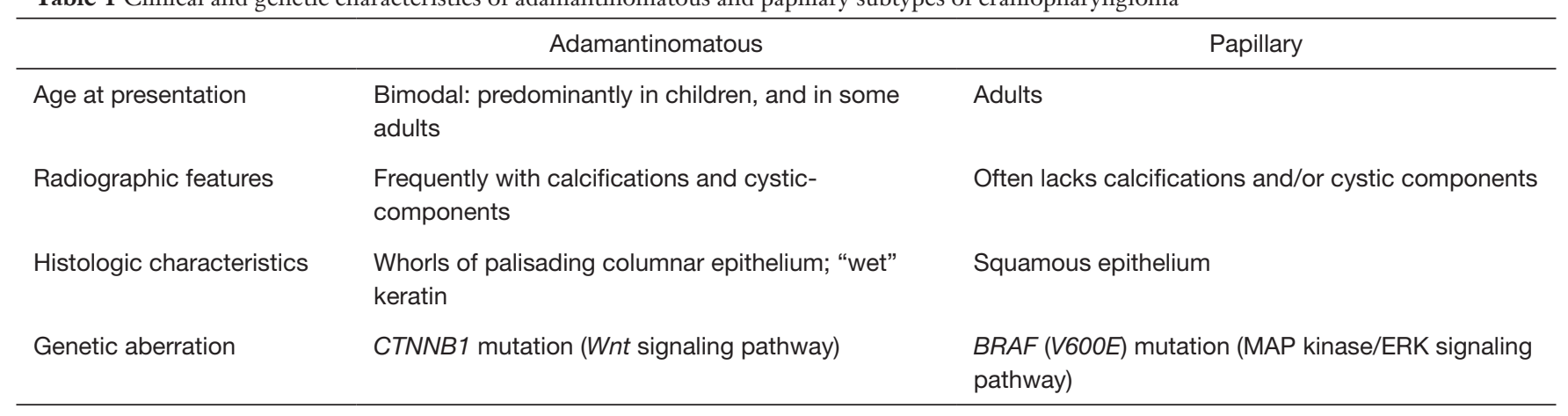

corresponded to variable clinical behavior of these tumors, and found that among five sites of somatic mutation there was no significant difference in progression-free survival; however, there was a trend towards poorer outcome amongst patients who harbored mutations at the Thr41 site though its significance was limited by small sample size (109). Guadagno et al. investigated markers that predicted increased risk of recurrence, and found that increased immunohistochemical expression tumor cell clusters containing $\beta$-catenin was associated with a higher rate of recurrence (110).

The presence of $C T T N B 1 / \beta$-catenin mutations that result in $W n t$ pathway activation has been corroborated as being a key marker in the pathogenesis and progression of adamantinomatous craniopharyngiomas, and studies are underway investigating this gene mutation as a target for therapeutic drug development (111). Furthermore, the discovery of $\beta$-catenin mutations as a principal factor in the clinical behavior of adamantinomatous craniopharyngiomas underscores the need for genetic testing and immunohistochemical staining to serve as important adjuncts in the pathologic diagnosis of these tumors as well as potentially predict clinical outcome. Although there are no clinical trials currently available for adamantinomatous craniopharyngiomas, several phase I and phase II trials are underway investigating $W n t / \beta$-catenin signaling inhibitors to treat other solid tumors (112), and the results of these studies may readily be translatable to craniopharyngiomas given the same underlying disease pathogenesis.

\section{BRAF (V600E) mutation in papillary craniopharyngioma}

Brastianos et al. first reported the presence of V600E BRAF oncogene mutation, with resultant activation of the MAP kinase/ERK signaling pathway, as a crucial underlying aberration that drives the pathogenesis of papillary craniopharyngiomas (113). Using whole exome sequencing analysis, V600E BRAF mutations were identified in all three 'discovery' papillary craniopharyngioma specimens (including one from a pediatric patient). None of these were found to harbor $C T N N B 1 / \beta$-catenin mutations. This finding was corroborated by performing targeted genotyping in a larger cohort, and BRAF mutations were detected in $95 \%$ of papillary craniopharyngiomas. Although it was already widely believed that the two subtypes of craniopharyngioma have distinct underlying genetic attributes, this study was the first to report the specific mutation driving the pathogenesis of the papillary subtype, thereby identifying a potential target for therapeutic drug treatment.

Since this crucial discovery, several case reports have been published that report a marked response to treatment of papillary craniopharyngiomas using BRAF inhibitors. Aylwin et al. were the first to report the successful treatment response of recurrent craniopharyngioma with the use of vemurafenib, a BRAF inhibitor. In their case report, a patient with histologically-confirmed papillary craniopharyngioma who had previously undergone three endoscopic tumor resections and adjuvant radiation therapy presented with tumor recurrence. The patient was treated with vemurafenib for three months, and repeat MRI showed a dramatic reduction in tumor size after two weeks. However, the tumor recurred within six weeks after treatment was discontinued (114). Brastianos et al. published a case report of a patient with recurrence of a large, cystic tumor who was treated with dual BRAF (dabrafenib) and MEK (trametinib) inhibitors, with resultant decrease of over $80 \%$ in the size of the tumor. Following this treatment, the patient's tumor was amenable to endoscopic resection, and with adjuvant radiosurgery, the authors report that he remained symptom-free 18 months after treatment (115). 
In another case study, Rostami et al. reported near resolution of tumor four months after initiating treatment with dual MEK and BRAF inhibitors. The treatment was discontinued after the patient developed pyrexia, and the subsequent clinical course, including whether tumor recurrence occurred, was not reported (116).

The BRAF inhibitor vemurafenib is currently FDAapproved for the treatment of late-stage melanoma, which frequently harbors the same V600E mutation as papillary craniopharyngioma. In one randomized phase III clinical trial, patients receiving vemurafenib had significantly longer overall survival compared to the chemotherapeutic agent dacarbazine (117). In another phase III trial for metastatic melanoma, a separate BRAF inhibitor (dabrafenib) and adjunct MEK inhibitor (trametinib) was shown to improve overall survival compared to BRAF inhibitor monotherapy, suggesting that the addition of a MEK inhibitor can protect against BRAF inhibitor resistance (118). However, the response of BRAF inhibitors to treat other types of tumors has been more guarded. Hyman et al. conducted a phase II study that investigated tumor response to vemurafenib in a variety of non-melanoma cancers harboring BRAF V600E mutations and found a highly variable tumor response. This study highlights that although targeted therapies can be developed to treat tumors harboring specific mutations, the sensitivity of response may vary between different types of tumors (119).

Given that there has been a singular underlying mutation identified that drives the pathogenesis of papillary craniopharyngiomas, the use of targeted drug therapy holds great promise for the treatment of this tumor. There is a phase II clinical trial currently underway (NCT03224767) investigating the combined use of BRAF and MEK-inhibitors (vemurafenib and cobimetinib) for the treatment of BRAF V600E mutant residual or recurrent craniopharyngiomas. Given the long-term morbidity associated with the current management paradigm for craniopharyngiomas, development of a targeted treatment for patients with BRAF V600E mutant papillary subtype tumors could serve not only for the treatment of recurrent tumors, but also potentially as a neoadjuvant therapy to reduce tumor size prior to surgery or radiation and limit the long-term morbidity associated with these treatments.

\section{Discussion}

There is a paucity of effective agents for refractory or relapsed meningioma, pituitary adenoma, or craniopharyngioma, though a new focus on investigating tumorigenic drivers has resulted in the development of promising therapies, particularly for $N F-2$ mutated meningiomas and craniopharyngiomas harboring mutations of V600E BRAF. In contrast, NF-2 wild-type meningiomas often exhibit a high mutational burden which may be more responsive to immunotherapy. Clarification of the genetic and epigenetic changes which drive development of pituitary adenomas is still developing, though the inverse relationship between MGMT immunoexpression and the response to temozolomide warrants interest.

\section{Acknowledgments}

The authors wish to acknowledge Somnath Das for his assistance with formatting this manuscript for publication. Funding: None.

\section{Footnote}

Provenance and Peer Review: This article was commissioned by the Guest Editor (Jon Glass) for the series "Targeted systemic therapies for primary and metastatic brain tumors" published in Chinese Clinical Oncology. The article was sent for external peer review organized by the Guest Editor and the editorial office.

Reporting Checklist: The authors have completed the Narrative Review reporting checklist. Available at http:// dx.doi.org/10.21037/cco-20-168

Conflicts of Interest: All authors have completed the ICMJE uniform disclosure form (available at http://dx.doi. org/10.21037/cco-20-168). The series "Targeted systemic therapies for primary and metastatic brain tumors" was commissioned by the editorial office without any funding or sponsorship. The authors have no other conflicts of interest to declare.

Ethical Statement: The authors are accountable for all aspects of the work in ensuring that questions related to the accuracy or integrity of any part of the work are appropriately investigated and resolved.

Open Access Statement: This is an Open Access article distributed in accordance with the Creative Commons Attribution-NonCommercial-NoDerivs 4.0 International License (CC BY-NC-ND 4.0), which permits the non- 
commercial replication and distribution of the article with the strict proviso that no changes or edits are made and the original work is properly cited (including links to both the formal publication through the relevant DOI and the license). See: https://creativecommons.org/licenses/by-nc-nd/4.0/.

\section{References}

1. Ostrom QT, Cioffi G, Gittleman H, et al. CBTRUS Statistical Report: Primary Brain and Other Central Nervous System Tumors Diagnosed in the United States in 2012-2016. Neuro Oncol 2019;21:v1-v100.

2. Cornelius JF, Slotty PJ, Steiger HJ, et al. Malignant potential of skull base versus non-skull base meningiomas: clinical series of 1,663 cases. Acta Neurochir (Wien) 2013;155:407-13.

3. Kalamarides M, Stemmer-Rachamimov AO, NiwaKawakita M, et al. Identification of a progenitor cell of origin capable of generating diverse meningioma histological subtypes. Oncogene 2011;30:2333-44.

4. Perry A, Stafford SL, Scheithauer BW, et al. Meningioma grading: an analysis of histologic parameters. Am J Surg Pathol 1997;21:1455-65.

5. Commins DL, Atkinson RD, Burnett ME. Review of meningioma histopathology. Neurosurg Focus 2007;23:E3.

6. Kleihues P, Cavenee WK, International Agency for Research on Cancer. Pathology and genetics of tumours of the nervous system. Lyon: IARC Press; 2000.

7. Lamszus K. Meningioma pathology, genetics, and biology. J Neuropathol Exp Neurol 2004;63:275-86.

8. Perry A, Gutmann DH, Reifenberger G. Molecular pathogenesis of meningiomas. J Neurooncol 2004;70:183-202.

9. Yang SY, Park CK, Park SH, et al. Atypical and anaplastic meningiomas: prognostic implications of clinicopathological features. J Neurol Neurosurg Psychiatry 2008;79:574-80.

10. Pasquier D, Bijmolt S, Veninga T, et al. Atypical and malignant meningioma: outcome and prognostic factors in 119 irradiated patients. A multicenter, retrospective study of the Rare Cancer Network. Int J Radiat Oncol Biol Phys 2008;71:1388-93.

11. Palma L, Celli P, Franco C, et al. Long-term prognosis for atypical and malignant meningiomas: a study of 71 surgical cases. J Neurosurg 1997;86:793-800.

12. Perry A, Scheithauer BW, Stafford SL, et al. "Malignancy" in meningiomas: a clinicopathologic study of 116 patients, with grading implications. Cancer 1999;85:2046-56.
13. Nanda A, Bir SC, Maiti TK, et al. Relevance of Simpson grading system and recurrence-free survival after surgery for World Health Organization Grade I meningioma. J Neurosurg 2017;126:201-11.

14. Marciscano AE, Stemmer-Rachamimov AO, Niemierko A, et al. Benign meningiomas (WHO Grade I) with atypical histological features: correlation of histopathological features with clinical outcomes. J Neurosurg 2016;124:106-14.

15. Park S, Cha YJ, Suh SH, et al. Risk group-adapted adjuvant radiotherapy for WHO grade I and II skull base meningioma. J Cancer Res Clin Oncol 2019;145:1351-60.

16. Korah MP, Nowlan AW, Johnstone PA, et al. Radiation therapy alone for imaging-defined meningiomas. Int J Radiat Oncol Biol Phys 2010;76:181-6.

17. Dziuk TW, Woo S, Butler EB, et al. Malignant meningioma: an indication for initial aggressive surgery and adjuvant radiotherapy. J Neurooncol 1998;37:177-88.

18. Chamberlain MC, Johnston SK. Hydroxyurea for recurrent surgery and radiation refractory meningioma: a retrospective case series. J Neurooncol 2011;104:765-71.

19. Ji Y, Rankin C, Grunberg S, et al. Double-Blind Phase III Randomized Trial of the Antiprogestin Agent Mifepristone in the Treatment of Unresectable Meningioma: SWOG S9005. J Clin Oncol 2015;33:4093-8.

20. Goodwin JW, Crowley J, Eyre HJ, et al. A phase II evaluation of tamoxifen in unresectable or refractory meningiomas: a Southwest Oncology Group study. J Neurooncol 1993;15:75-7.

21. Simó M, Argyriou AA, Macia M, et al. Recurrent highgrade meningioma: a phase II trial with somatostatin analogue therapy. Cancer Chemother Pharmacol 2014;73:919-23.

22. Norden AD, Ligon KL, Hammond SN, et al. Phase II study of monthly pasireotide LAR (SOM230C) for recurrent or progressive meningioma. Neurology 2015;84:280-6.

23. Chamberlain MC, Tsao-Wei DD, Groshen S. Temozolomide for treatment-resistant recurrent meningioma. Neurology 2004;62:1210-2.

24. Chamberlain MC. Adjuvant combined modality therapy for malignant meningiomas. J Neurosurg 1996;84:733-6.

25. Rouleau GA, Merel P, Lutchman M, et al. Alteration in a new gene encoding a putative membrane-organizing protein causes neuro-fibromatosis type 2 . Nature 1993;363:515-21.

26. Sahm F, Schrimpf D, Stichel D, et al. DNA methylationbased classification and grading system for meningioma: 
a multicentre, retrospective analysis. Lancet Oncol 2017;18:682-94.

27. Lamszus K, Lengler U, Schmidt NO, et al. Vascular endothelial growth factor, hepatocyte growth factor/ scatter factor, basic fibroblast growth factor, and placenta growth factor in human meningiomas and their relation to angiogenesis and malignancy. Neurosurgery 2000;46:93847; discussion 947-8.

28. Panagopoulos AT, Lancellotti CL, Veiga JC, et al. Expression of cell adhesion proteins and proteins related to angiogenesis and fatty acid metabolism in benign, atypical, and anaplastic meningiomas. J Neurooncol 2008;89:73-87.

29. Preusser M, Hassler M, Birner P, et al.

Microvascularization and expression of VEGF and its receptors in recurring meningiomas: pathobiological data in favor of anti-angiogenic therapy approaches. Clin Neuropathol 2012;31:352-60.

30. Nassehi D, Dyrbye H, Andresen M, et al. Vascular endothelial growth factor A protein level and gene expression in intracranial meningiomas with brain edema. APMIS 2011;119:831-43.

31. Furtner J, Schopf V, Seystahl K, et al. Kinetics of tumor size and peritumoral brain edema before, during, and after systemic therapy in recurrent WHO grade II or III meningioma. Neuro Oncol 2016;18:401-7.

32. Shih KC, Chowdhary S, Rosenblatt P, et al. A phase II trial of bevacizumab and everolimus as treatment for patients with refractory, progressive intracranial meningioma. J Neurooncol 2016;129:281-8.

33. Mauro A, Di Sapio A, Mocellini C, et al. Control of meningioma cell growth by platelet-derived growth factor (PDGF). J Neurol Sci 1995;131:135-43.

34. Maxwell M, Galanopoulos T, Hedley-Whyte ET, et al. Human meningiomas co-express platelet-derived growth factor (PDGF) and PDGF-receptor genes and their protein products. Int J Cancer 1990;46:16-21.

35. Wang JL, Nister M, Hermansson M, et al. Expression of PDGF beta-receptors in human meningioma cells. Int J Cancer 1990;46:772-8.

36. Shamah SM, Alberta JA, Giannobile WV, et al. Detection of activated platelet-derived growth factor receptors in human meningioma. Cancer Res 1997;57:4141-7.

37. Wen PY, Yung WK, Lamborn KR, et al. Phase II study of imatinib mesylate for recurrent meningiomas (North American Brain Tumor Consortium study 01-08). Neuro Oncol 2009;11:853-60.

38. Reardon DA, Norden AD, Desjardins A, et al. Phase II study of Gleevec(R) plus hydroxyurea (HU) in adults with progressive or recurrent meningioma. J Neurooncol 2012;106:409-15.

39. Kaley TJ, Wen P, Schiff D, et al. Phase II trial of sunitinib for recurrent and progressive atypical and anaplastic meningioma. Neuro Oncol 2015;17:116-21.

40. Raizer JJ, Grimm SA, Rademaker A, et al. A phase II trial of PTK787/ZK 222584 in recurrent or progressive radiation and surgery refractory meningiomas. J Neurooncol 2014;117:93-101.

41. Kros J, de Greve K, van Tilborg A, et al. NF2 status of meningiomas is associated with tumour localization and histology. J Pathol 2001;194:367-72.

42. James MF, Han S, Polizzano C, Plotkin SR, et al. NF2/ merlin is a novel negative regulator of mTOR complex 1 , and activation of mTORC1 is associated with meningioma and schwannoma growth. Mol Cell Biol 2009;29:4250-61.

43. James MF, Stivison E, Beauchamp R, et al. Regulation of mTOR complex 2 signaling in neurofibromatosis 2-deficient target cell types. Mol Cancer Res 2012;10:649-59.

44. Bi WL, Greenwald NF, Abedalthagafi M, et al. Genomic landscape of high-grade meningiomas. NPJ Genom Med 2017;2:15.

45. Hao S, Huang G, Feng J, et al. Non-NF2 mutations have a key effect on inhibitory immune checkpoints and tumor pathogenesis in skull base meningiomas. J Neurooncol 2019;144:11-20.

46. Boetto J, Apra C, Bielle F, et al. Selective vulnerability of the primitive meningeal layer to prenatal Smo activation for skull base meningothelial meningioma formation. Oncogene 2018;37:4955-63.

47. Clark VE, Harmanci AS, Bai H, et al. Recurrent somatic mutations in POLR2A define a distinct subset of meningiomas. Nat Genet 2016;48:1253-9.

48. Abedalthagafi M, Bi WL, Aizer AA, et al. Oncogenic PI3K mutations are as common as AKT1 and SMO mutations in meningioma. Neuro Oncol 2016;18:649-55.

49. Brastianos PK, Horowitz PM, Santagata S, et al. Genomic sequencing of meningiomas identifies oncogenic SMO and AKT1 mutations. Nat Genet 2013;45:285-9.

50. Boetto J, Bielle F, Sanson M, et al. SMO mutation status defines a distinct and frequent molecular subgroup in olfactory groove meningiomas. Neuro Oncol 2017;19:345-51.

51. Yesilöz Ü, Kirches E, Hartmann C, et al. Frequent AKT1E17K mutations in skull base meningiomas are associated with $\mathrm{mTOR}$ and ERK1/2 activation and reduced time to tumor recurrence. Neuro Oncol 2017;19:1088-96. 
52. Hyman DM, Smyth LM, Donoghue MTA, et al. AKT Inhibition in Solid Tumors With AKT1 Mutations. J Clin Oncol 2017;35:2251-9.

53. Weller M, Roth P, Sahm F, et al. Durable Control of Metastatic AKT1-Mutant WHO Grade 1 Meningothelial Meningioma by the AKT Inhibitor, AZD5363. J Natl Cancer Inst 2017;109:1-4.

54. Clark VE, Erson-Omay EZ, Serin A, et al. Genomic analysis of non-NF2 meningiomas reveals mutations in TRAF7, KLF4, AKT1, and SMO. Science 2013;339:1077-80.

55. Du Z, Abedalthagafi M, Aizer AA, et al. Increased expression of the immune modulatory molecule PDL1 (CD274) in anaplastic meningioma. Oncotarget 2015;6:4704-16.

56. Fang L, Lowther DE, Meizlish ML, et al. The immune cell infiltrate populating meningiomas is composed of mature, antigen-experienced T and B cells. Neuro Oncol 2013;15:1479-90.

57. Dunn IF, Du Z, Touat M, et al. Mismatch repair deficiency in high-grade meningioma: a rare but recurrent event associated with dramatic immune activation and clinical response to PD-1 blockade. JCO Precis Oncol 2018;2018:10.1200/PO.18.00190.

58. Roelfsema F, Biermasz NR, Pereira AM. Clinical factors involved in the recurrence of pituitary adenomas after surgical remission: a structured review and meta-analysis. Pituitary 2012;15:71-83.

59. Cappabianca P, Solari D. The endoscopic endonasal approach for the treatment of recurrent or residual pituitary adenomas: widening what to see expands what to do? World Neurosurg 2012;77:455-6.

60. Cavallo LM, Solari D, Tasiou A, et al. Endoscopic endonasal transsphenoidal removal of recurrent and regrowing pituitary adenomas: experience on a 59-patient series. World Neurosurg 2013;80:342-50.

61. Chang EF, Zada G, Kim S, et al. Long-term recurrence and mortality after surgery and adjuvant radiotherapy for nonfunctional pituitary adenomas. J Neurosurg 2008;108:736-45.

62. Rosen MR, Saigal K, Evans J, et al. A review of the endoscopic approach to the pituitary through the sphenoid sinus. Curr Opin Otolaryngol Head Neck Surg 2006;14:6-13.

63. Sheehan JP, Xu Z, Lobo MJ. External beam radiation therapy and stereotactic radiosurgery for pituitary adenomas. Neurosurg Clin N Am 2012;23:571-86.

64. Raverot G, Burman P, McCormack A, et al. European
Society of Endocrinology Clinical Practice Guidelines for the management of aggressive pituitary tumours and carcinomas. Eur J Endocrinol 2018;178:G1-G24.

65. Lopes MBS. The 2017 World Health Organization classification of tumors of the pituitary gland: a summary. Acta Neuropathol 2017;134:521-35.

66. Mete O, Lopes MB. Overview of the 2017 WHO Classification of Pituitary Tumors. Endocr Pathol 2017;28:228-43.

67. Pernicone PJ, Scheithauer BW, Sebo TJ, et al. Pituitary carcinoma: a clinicopathologic study of 15 cases. Cancer 1997;79:804-12.

68. Lamb LS, Sim HW, McCormack AI. Exploring the Role of Novel Medical Therapies for Aggressive Pituitary Tumors: A Review of the Literature-"Are We There Yet?". Cancers (Basel) 2020;12:308.

69. McCormack A, Dekkers OM, Petersenn S, et al. Treatment of aggressive pituitary tumours and carcinomas: results of a European Society of Endocrinology (ESE) survey 2016. Eur J Endocrinol 2018;178:265-76.

70. Caimari F, Korbonits M. Novel Genetic Causes of Pituitary Adenomas. Clin Cancer Res 2016;22:5030-42.

71. Chen J, Jian X, Deng S, et al. Identification of recurrent USP48 and BRAF mutations in Cushing's disease. Nat Commun 2018;9:3171.

72. Fadul CE, Kominsky AL, Meyer LP, et al. Long-term response of pituitary carcinoma to temozolomide. Report of two cases. J Neurosurg 2006;105:621-6.

73. Lim S, Shahinian H, Maya MM, et al. Temozolomide: a novel treatment for pituitary carcinoma. Lancet Oncol 2006;7:518-20.

74. Ilie MD, Lasolle H, Raverot G. Emerging and Novel Treatments for Pituitary Tumors. J Clin Med 2019;8:1107.

75. Ortiz LD, Syro LV, Scheithauer BW, et al. Anti-VEGF therapy in pituitary carcinoma. Pituitary 2012;15:445-9.

76. Wang Y, He Q, Meng X, et al. Apatinib (YN968D1) and temozolomide in recurrent invasive pituitary adenoma: case report and literature review. World Neurosurg 2019. [Epub ahead of print]. doi: 10.1016/j.wneu.2018.12.174.

77. Dworakowska D, Wlodek E, Leontiou CA, et al. Activation of RAF/MEK/ERK and PI3K/AKT/mTOR pathways in pituitary adenomas and their effects on downstream effectors. Endocr Relat Cancer 2009;16:1329-38.

78. Jouanneau E, Wierinckx A, Ducray F, et al. New targeted therapies in pituitary carcinoma resistant to temozolomide. Pituitary 2012;15:37-43.

79. Wang PF, Wang TJ, Yang YK, et al. The expression profile of PD-L1 and CD8(+) lymphocyte in pituitary 
adenomas indicating for immunotherapy. J Neurooncol 2018;139:89-95.

80. Mei Y, Bi WL, Greenwald NF, et al. Increased expression of programmed death ligand 1 (PD-L1) in human pituitary tumors. Oncotarget 2016;7:76565-76.

81. Lin AL, Jonsson P, Tabar V, et al. Marked Response of a Hypermutated ACTH-Secreting Pituitary Carcinoma to Ipilimumab and Nivolumab. J Clin Endocrinol Metab 2018;103:3925-30.

82. Albarel F, Gaudy C, Castinetti F, et al. Long-term followup of ipilimumab-induced hypophysitis, a common adverse event of the anti-CTLA-4 antibody in melanoma. Eur J Endocrinol 2015;172:195-204.

83. Faje AT, Sullivan R, Lawrence D, et al. Ipilimumabinduced hypophysitis: a detailed longitudinal analysis in a large cohort of patients with metastatic melanoma. J Clin Endocrinol Metab 2014;99:4078-85.

84. Iwama S, De Remigis A, Callahan MK, et al. Pituitary expression of CTLA-4 mediates hypophysitis secondary to administration of CTLA-4 blocking antibody. Sci Transl Med 2014;6:230ra45.

85. Jane JA Jr, Laws ER. Craniopharyngioma. Pituitary 2006;9:323-6.

86. Bunin GR, Surawicz TS, Witman PA, et al. The descriptive epidemiology of craniopharyngioma. J Neurosurg 1998;89:547-51.

87. Larkin SJ, Ansorge O. Pathology and pathogenesis of craniopharyngiomas. Pituitary 2013;16:9-17.

88. Curran JG, O'Connor E. Imaging of craniopharyngioma. Childs Nerv Syst 2005;21:635-9.

89. Müller HL, Merchant TE, Puget S, et al. New outlook on the diagnosis, treatment and follow-up of childhood-onset craniopharyngioma. Nat Rev Endocrinol 2017;13:299-312.

90. Boekhoff S, Bison B, Eveslage M, et al. Craniopharyngiomas presenting as incidentalomas: results of KRANIOPHARYNGEOM 2007. Pituitary 2019;22:532-41.

91. Lopez-Serna R, Gomez-Amador JL, Barges-Coll $\mathrm{J}$, et al. Treatment of craniopharyngioma in adults: systematic analysis of a 25-year experience. Arch Med Res 2012;43:347-55.

92. Fahlbusch R, Honegger J, Paulus W, et al. Surgical treatment of craniopharyngiomas: experience with 168 patients. J Neurosurg 1999;90:237-50.

93. Komotar RJ, Starke RM, Raper DM, et al. Endoscopic endonasal compared with microscopic transsphenoidal and open transcranial resection of craniopharyngiomas. World Neurosurg 2012;77:329-41.
94. Rajan B, Ashley S, Gorman C, et al. Craniopharyngioma-a long-term results following limited surgery and radiotherapy. Radiother Oncol 1993;26:1-10.

95. Scott RM, Hetelekidis S, Barnes PD, et al. Surgery, radiation, and combination therapy in the treatment of childhood craniopharyngioma--a 20-year experience. Pediatr Neurosurg 1994;21 Suppl 1:75-81.

96. Yang I, Sughrue ME, Rutkowski MJ, et al. Craniopharyngioma: a comparison of tumor control with various treatment strategies. Neurosurg Focus 2010;28:E5.

97. Combs SE, Thilmann C, Huber PE, et al. Achievement of long-term local control in patients with craniopharyngiomas using high precision stereotactic radiotherapy. Cancer 2007;109:2308-14.

98. Fitzek MM, Linggood RM, Adams J, Munzenrider JE. Combined proton and photon irradiation for craniopharyngioma: long-term results of the early cohort of patients treated at Harvard Cyclotron Laboratory and Massachusetts General Hospital. Int J Radiat Oncol Biol Phys 2006;64:1348-54.

99. Habrand JL, Ganry O, Couanet D, et al. The role of radiation therapy in the management of craniopharyngioma: a 25-year experience and review of the literature. Int J Radiat Oncol Biol Phys 1999;44:255-63.

100. Karavitaki N, Cudlip S, Adams CB, et al. Craniopharyngiomas. Endocr Rev 2006;27:371-97.

101. Moon SH, Kim IH, Park SW, et al. Early adjuvant radiotherapy toward long-term survival and better quality of life for craniopharyngiomas--a study in single institute. Childs Nerv Syst 2005;21:799-807.

102.Zada G, Kintz N, Pulido M, et al. Prevalence of neurobehavioral, social, and emotional dysfunction in patients treated for childhood craniopharyngioma: a systematic literature review. PLoS One 2013;8:e76562.

103. Gautier A, Godbout A, Grosheny C, et al. Markers of recurrence and long-term morbidity in craniopharyngioma: a systematic analysis of 171 patients. J Clin Endocrinol Metab 2012;97:1258-67.

104. Malgulwar PB, Nambirajan A, Pathak P, et al. Study of beta-catenin and BRAF alterations in adamantinomatous and papillary craniopharyngiomas: mutation analysis with immunohistochemical correlation in 54 cases. J Neurooncol 2017;133:487-95.

105. Brembeck FH, Rosario M, Birchmeier W. Balancing cell adhesion and Wnt signaling, the key role of beta-catenin. Curr Opin Genet Dev 2006;16:51-9.

106. Morin PJ. beta-catenin signaling and cancer. Bioessays 1999;21:1021-30. 
107.Martinez-Barbera JP, Buslei R. Adamantinomatous craniopharyngioma: pathology, molecular genetics and mouse models. J Pediatr Endocrinol Metab 2015;28:7-17.

108. Sekine S, Shibata T, Kokubu A, et al. Craniopharyngiomas of adamantinomatous type harbor beta-catenin gene mutations. Am J Pathol 2002;161:1997-2001.

109. Goschzik T, Gessi M, Dreschmann V, Gebhardt U, et al. Genomic Alterations of Adamantinomatous and Papillary Craniopharyngioma. J Neuropathol Exp Neurol 2017;76:126-34.

110. Guadagno E, de Divitiis O, Solari D, et al. Can recurrences be predicted in craniopharyngiomas? betacatenin coexisting with stem cells markers and p-ATM in a clinicopathologic study of 45 cases. J Exp Clin Cancer Res 2017;36:95.

111. Anastas JN, Moon RT. WNT signalling pathways as therapeutic targets in cancer. Nat Rev Cancer 2013;13:11-26.

112.Jung YS, Park JI. Wnt signaling in cancer: therapeutic targeting of Wnt signaling beyond beta-catenin and the destruction complex. Exp Mol Med 2020;52:183-91.

113. Brastianos PK, Taylor-Weiner A, Manley PE, et al.

Cite this article as: Martinez NL, Khanna O, Farrell CJ. A narrative review of targeted therapy in meningioma, pituitary adenoma, and craniopharyngioma of the skull base. Chin Clin Oncol 2020;9(6):75. doi: 10.21037/cco-20-168
Exome sequencing identifies BRAF mutations in papillary craniopharyngiomas. Nat Genet 2014;46:161-5.

114. Aylwin SJ, Bodi I, Beaney R. Pronounced response of papillary craniopharyngioma to treatment with vemurafenib, a BRAF inhibitor. Pituitary 2016;19:544-6.

115. Brastianos PK, Shankar GM, Gill CM, et al. Dramatic Response of BRAF V600E Mutant Papillary Craniopharyngioma to Targeted Therapy. J Natl Cancer Inst 2015;108:djv310 .

116. Rostami E, Witt Nystrom P, Libard S, et al. Recurrent papillary craniopharyngioma with BRAFV600E mutation treated with neoadjuvant-targeted therapy. Acta Neurochir (Wien) 2017;159:2217-21.

117. Chapman PB, Hauschild A, Robert C, et al. Improved survival with vemurafenib in melanoma with BRAF V600E mutation. N Engl J Med 2011;364:2507-16.

118. Robert C, Karaszewska B, Schachter J, et al. Improved overall survival in melanoma with combined dabrafenib and trametinib. N Engl J Med 2015;372:30-9.

119. Hyman DM, Puzanov I, Subbiah V, et al. Vemurafenib in Multiple Nonmelanoma Cancers with BRAF V600 Mutations. N Engl J Med 2015;373:726-36. 